\title{
Pastiches votifs à Rome
}

Du Pasquin à Ex voto fecit

\section{Anne Lepoittevin}

\section{(2) OpenEdition \\ Journals}

Édition électronique

URL : https://journals.openedition.org/tc/9925

DOI : $10.4000 /$ tc. 9925

ISSN : 1952-420X

Éditeur

Éditions de l'EHESS

\section{Édition imprimée}

Date de publication : 30 octobre 2018

Pagination : 220-239

ISBN : 2-7132-2751-6

ISSN : 0248-6016

Référence électronique

Anne Lepoittevin, "Pastiches votifs à Rome», Techniques \& Culture [En ligne], 70 | 2018, mis en ligne le 06 décembre 2020, consulté le 29 septembre 2022. URL : http://journals.openedition.org/tc/9925 ;

DOI : https://doi.org/10.4000/tc.9925 


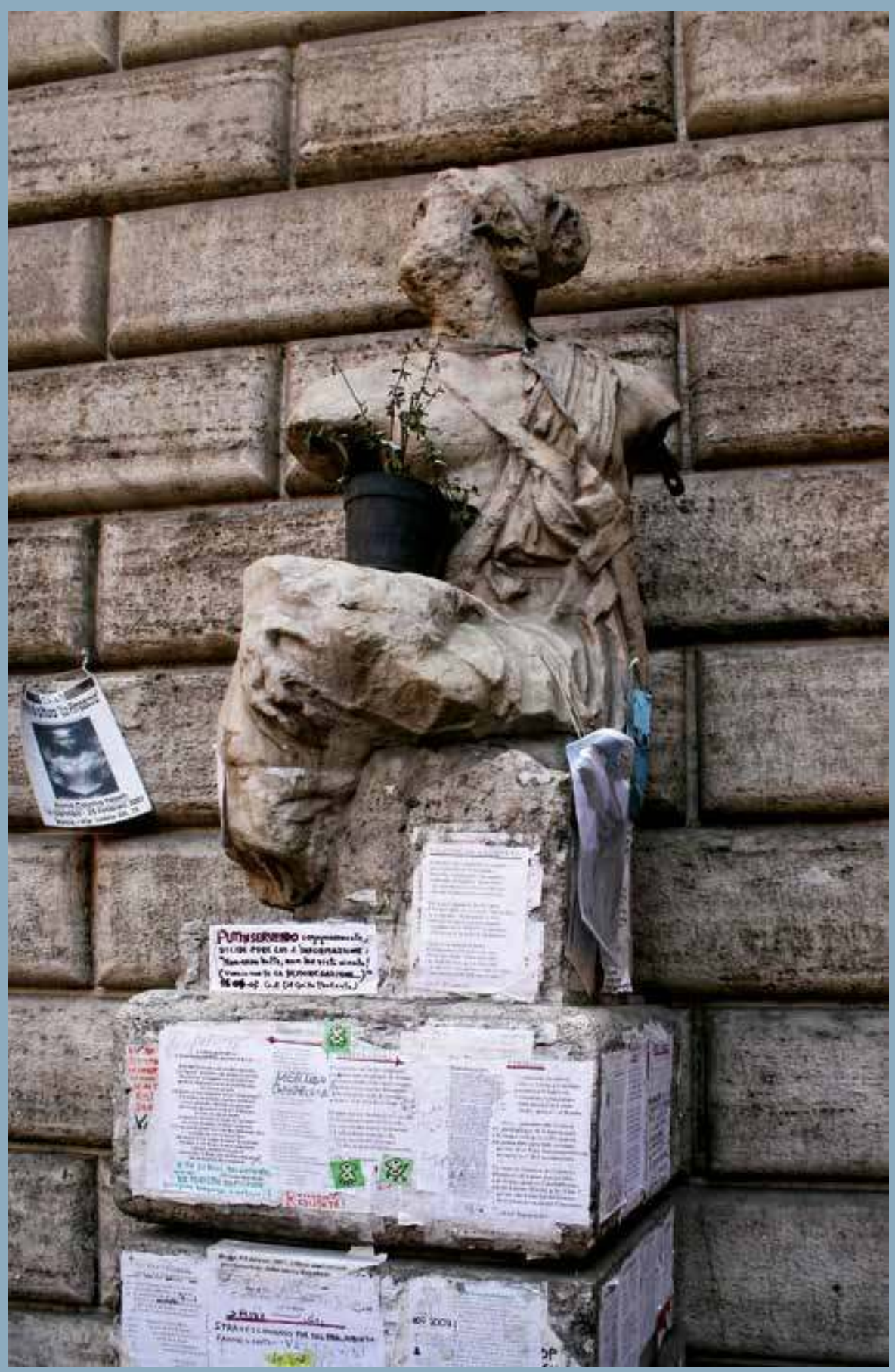




\section{Pastiches votifs à Rome}

\section{Du Pasquin à Ex voto fecit}

Les moyens de reproduction modernes ayant d'une part allégé les temps et coûts de production, de l'autre facilité la copie, le collage et la modification des images, pastiches et caricatures se sont répandus entre $\mathrm{xvI}^{\mathrm{e}}$ et $\mathrm{xx}$ siècles, avant de proliférer avec l'image numérique. Reste que le succès d'un pastiche ne repose pas sur une manipulation technique, mais sur un jeu avec un code surdéterminé par le lieu et l'époque, car le public doit pouvoir l'identifier au premier coup d'œil. On comprendra alors l'efficacité des pastiches d'art religieux à Rome à travers les siècles. Parmi ceux-ci, les pastiches votifs empruntent leurs codes et leur langage au dispositif votif catholique, omniprésent dans la Ville: ils associent systématiquement une image miraculeuse, souvent mariale, valorisée dans le sanctuaire et/ou dans l'espace urbain, et des objets et des textes votifs témoignant du pouvoir d'intercession du prototype représenté. Rare pour les périodes anciennes, la parodie votive est aujourd'hui extrêmement fréquente, à l'instar des multiples emprunts et détournements de l'art religieux qui caractérisent l'art contemporain romain. Une gravure moderne et des affiches contemporaines permettront de réfléchir aux détournements du dispositif votif ${ }^{1}$, efficaces à des fins avant tout politiques, à une période où le politique est - ô combien - religieux (la Rome du Papa-Rè), et à la nôtre où il le reste partiellement, à l'ombre du Cupolone.

\section{Pasquin et les statues parlantes}

Dès le début du xvie siècle, les Romains ont placardé des libelles sur six statues placées dans les rues de leur ville, Pasquino, Marforio, Madama Lucrezia, l'Abate Luigi, il Babbuino et il Facchino. Critiquant ouvertement le pouvoir pontifical, ces statues parlantes, constituées en 
personnages, ont prospéré sur un terrain indissociablement politique et religieux. Ceci vaut pour la teneur des critiques adressées à la hiérarchie ecclésiastique, mais également pour les formes d'agency, largement empruntées aux images miraculeuses chrétiennes (Sansterre 2016), que les Romains ont conférées à ces médiums, leurs intercesseurs auprès des plus hautes instances.

Ainsi Pasquin, la plus célèbre et la plus séditieuse des six statues, détourne-t-il intentionnellement certains agencements cultuels, parmi lesquels la prière et l'ex-voto. De tels détournements ont été facilités par le manque de lisibilité de l'œuvre, un groupe antique de main inconnue dont on discute encore la date et le sujet: s'agit-il d'un marbre hellénistique ou d'une copie romaine? de Mélénas soulevant Patrocle mourant ou d'Ajax portant le cadavre d'Achille (Michel dAnnoville 2016: 1) ? Seule son histoire moderne est bien connue à compter de sa redécouverte en - ou avant - 1501 dans les fouilles de la place Navone. Dès 1501, le marbre a été placé sur un piédestal au coin du Palais Orsini, aujourd'hui Palais Braschi - un emplacement qu'aurait aussi bien pu occuper l'une des nombreuses Vierges à l'Enfant peintes et sculptées qui veillent sur les rues de la ville. Sa position dans l'Urbs est stratégique car le groupe statuaire se trouve à proximité de la via Papalis qui unit Saint-Pierre au Latran. La vie sociale et civique y bat son plein, le tourisme profane et religieux y est dense. Ainsi entouré, Pasquin est immédiatement devenu un personnage, et sa personnification a fait oublier le torse du second personnage ainsi que le sujet héroïque du groupe d'origine. Il a d'abord été associé à la fête de saint Marc célébrée le 25 avril dans la basilique voisine de San Lorenzo in Damaso : déguisé, il « dit» des épigrammes composées pour l'occasion ${ }^{2}$. Dès les années 1510, le marbre s'exprime toute l'année. Il devient alors « un lieu cultuel spécifique », le porte-parole d'une communauté, les habitants de Rome, qui proteste contre le pape en latin, en dialecte romain et en toscan. Aujourd'hui encore, on y dépose en cachette de nombreux écrits contre la municipalité, l'État et le Vatican, et parfois de modestes dons, des colliers, des fleurs ${ }^{3}$.

La verve du marbre est allée croissant au $\mathrm{XvI}^{\mathrm{e}}$ siècle, et Pasquin a eu tôt fait de parodier des modèles empruntés au discours religieux. Dès les années 1510, il s'en prend aux papes, seul ou dans ses dialogues avec ses collègues, notamment le marbre romain Marforio (Rome, Musées capitolins). Et, s'il s'adresse à tous les puissants (le Christ, Marie, saint Pierre, les papes, les cardinaux, tel grand prélat, Charles Quint, François I I ${ }^{\text {, }}$ les ambassadeurs...), c'est pour les apostropher sur la situation romaine. Irrévérencieux, il contrefait souvent les Écritures et les prières: ainsi du "Gloria in Excelsis Deo. Di Carolo Quinto Imperatore Romano», de «l'Ave Maria di Mastro Pasquino sopra papa Paol Terzo», du «Miserere Mei», beaucoup de ces textes ayant été déposés devant la statue avant de connaître une postérité littéraire (Catelli 2008: 19 n. 3). Inlassable, il dénonce la corruption des pontifes et de l'ensemble du clergé, leur amour de l'or, la rupture de leurs vœux, le scandale des indulgences, le culte des objets, les impôts pesant sur le peuple. Dès cette période, les pasquinades franchissent les murs de Rome. Rien d'étonnant dès lors à ce qu'elles portent la parole protestante à partir des années 1520, et davantage encore dans les années 1540 (Lastraioli 2014, Damianaki-Romano 2014), poursuivant les critiques réactivées par les réformés ou mentionnant directement Luther ${ }^{4}$. S'adressant à Pasquin, saint Pierre en 
personne menace de déménager dans les Flandres. La satire protestante donne au Pasquin une audience extraordinaire en France, en Espagne et dans les régions gagnées à la Réforme.

Si les sources littéraires témoignent largement du détournement des modèles religieux appliqués au Pasquin, les sources visuelles sont en reste, à l'exception d'une gravure anonyme du Pasquin montrant la contrefaçon d'un dispositif votif, absolument remarquable en raison de l'époque (le second $\mathrm{xvI}^{\mathrm{e}}$ siècle), du lieu (Rome) et du réseau de diffusion (antiquaire) auquel la planche était promise. Dès la première moitié du Xvile siècle, la réputation du marbre avait commencé d'emprunter les réseaux antiquaires. Les gravures rendant compte des merveilles de Rome apparaissent dans les années 1520, et se multiplient dans les années 1540 et 1550 (Zorach 2008: 32-34). Le Speculum Romanae Magnificentiae d'Antoine Lafréry (ca 1512-1577), dont est extraite la gravure de Pasquin, constitue le meilleur exemple de ce phénomène (Parshall 2006). Lafréry, un graveur français arrivé à Rome au début des années 1540, propose aux touristes et aux amateurs des vues des monuments et des statues antiques et modernes de la Ville. Ceux-ci les sélectionnent à leur guise, et Lafréry les assemble derrière le frontispice du Speculum Magnificentiae Romae (ibid.: 11-24). Dès 1550, la gravure du Pasquin est disponible dans son échoppe (Karmon 2008: 46). Souvent publiée, cette œuvre n’a pas été commentée en raison de son iconographie, inhabituelle. Si les textes rapportés par la gravure ne critiquent pas frontalement le pape, la construction de l'image parodie bel et bien un dispositif votif. Cette parodie pouvait sembler aisée tant Pasquin présentait de caractéristiques communes aux statues miraculeuses: ancien et de main inconnue, il était placé en hauteur, visible de tous, vêtu en fonction du calendrier, parlant aux puissants, et entouré de nombreux écrits réputés efficaces.

Cadrage, textes et objets visibles sur la gravure concourent à présenter Pasquin comme une statue miraculeuse et les différents textes et objets qui l'entourent comme des ex-voto. Cadrage et textes insistent sur la fonction d'intercesseur d'un Pasquin presque écorché5: il regarde le ciel, bouche ouverte. Plus spécifiquement, les textes, qui encerclent la figure principale sur trois côtés, revendiquent le statut divin du personnage ainsi que ses différentes facultés ${ }^{6}$. Dans un quatrain situé à hauteur de son genou, Pasquin explique que sa statue présente sa vraie image: «non conoscete la vera effigie mia?» (« Ne connaissez-vous point ma vraie image?»). La référence à la Véronique, «la vera effigie mia», maltraitée pendant le sac de Rome de 1527 (Chastel 1984 : 143-44), la place au nombre des images acheiropoïètes. Et les deux vers suivants «Io son Pasquin col mal che Dio vi dia / Vi possa venir i strangoglioni» ("Je suis Pasquin et avec le sort que Dieu vous jette/ Puissent vous venir les estranguillons») explicitent le lien entre Dieu, Pasquin et la communauté de ses adorateurs. Assonances et voisinages, «io/vi »// dio/dia» (moi/vous//dieu/ donne) associent « dieu », «moi » et «Dieu qui vous donne» à ma demande. Le bienfait procuré étant une maladie qui vient aux équidés et donc aux ânes, les estranguillons, comme si Pasquin souhaitait la mort à ses courtisans. Car, victime de son succès, et misanthrope, il se plaint de l'affluence: "Che fate qui a torno babioni » / "Pourquoi venir ici, tas de babouins ${ }^{7}$ ? » Tout en reprenant ailleurs un "vobis ego ${ }^{8}$ », parodie d'antiphonaire, instaurant un lien de nature divine entre Pasquin et la foule. 
Pasquin en gravure. Anonyme, 1550. Extrait d'Antoine Lafréry, Speculum Romanae Magnificentiae

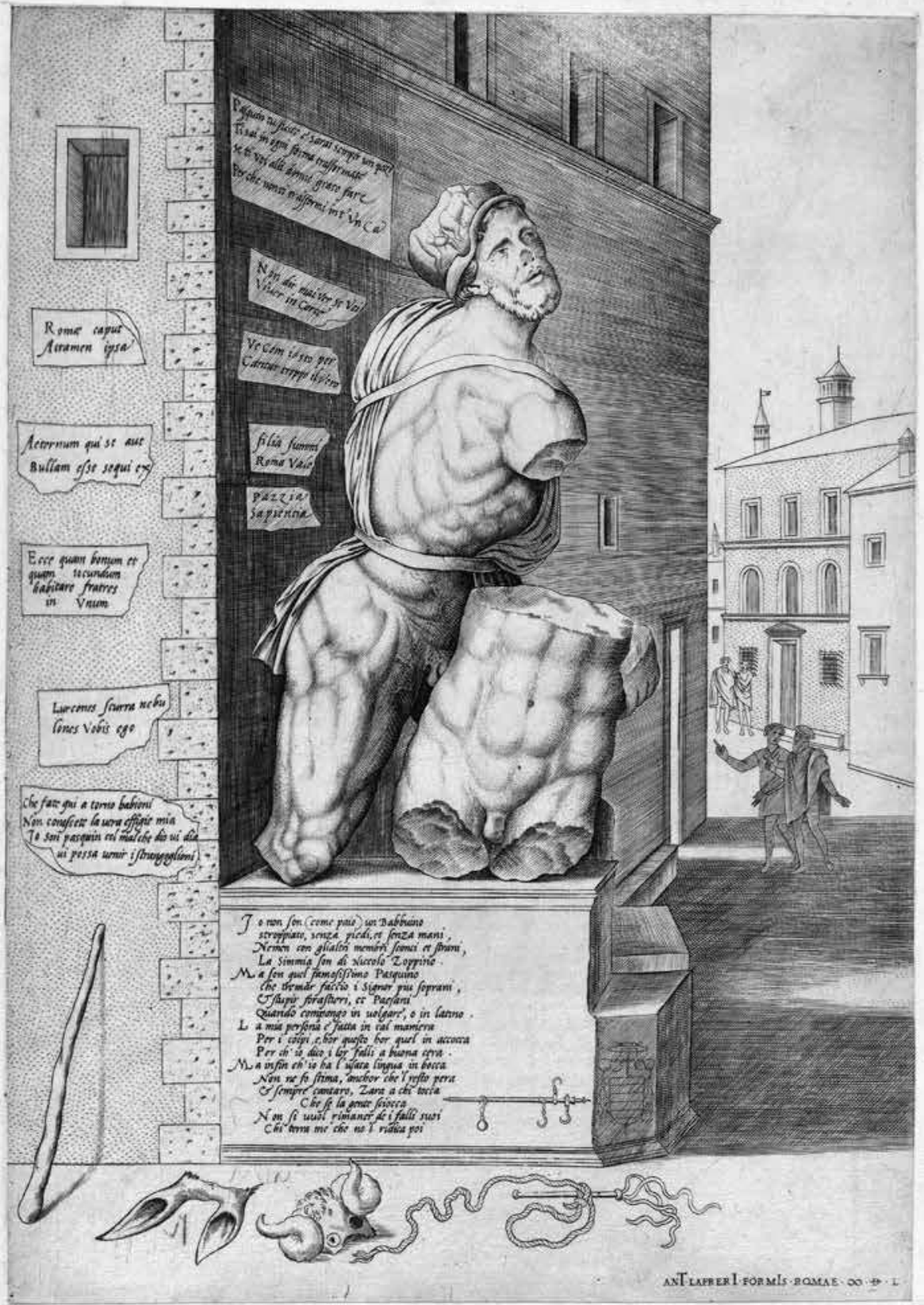




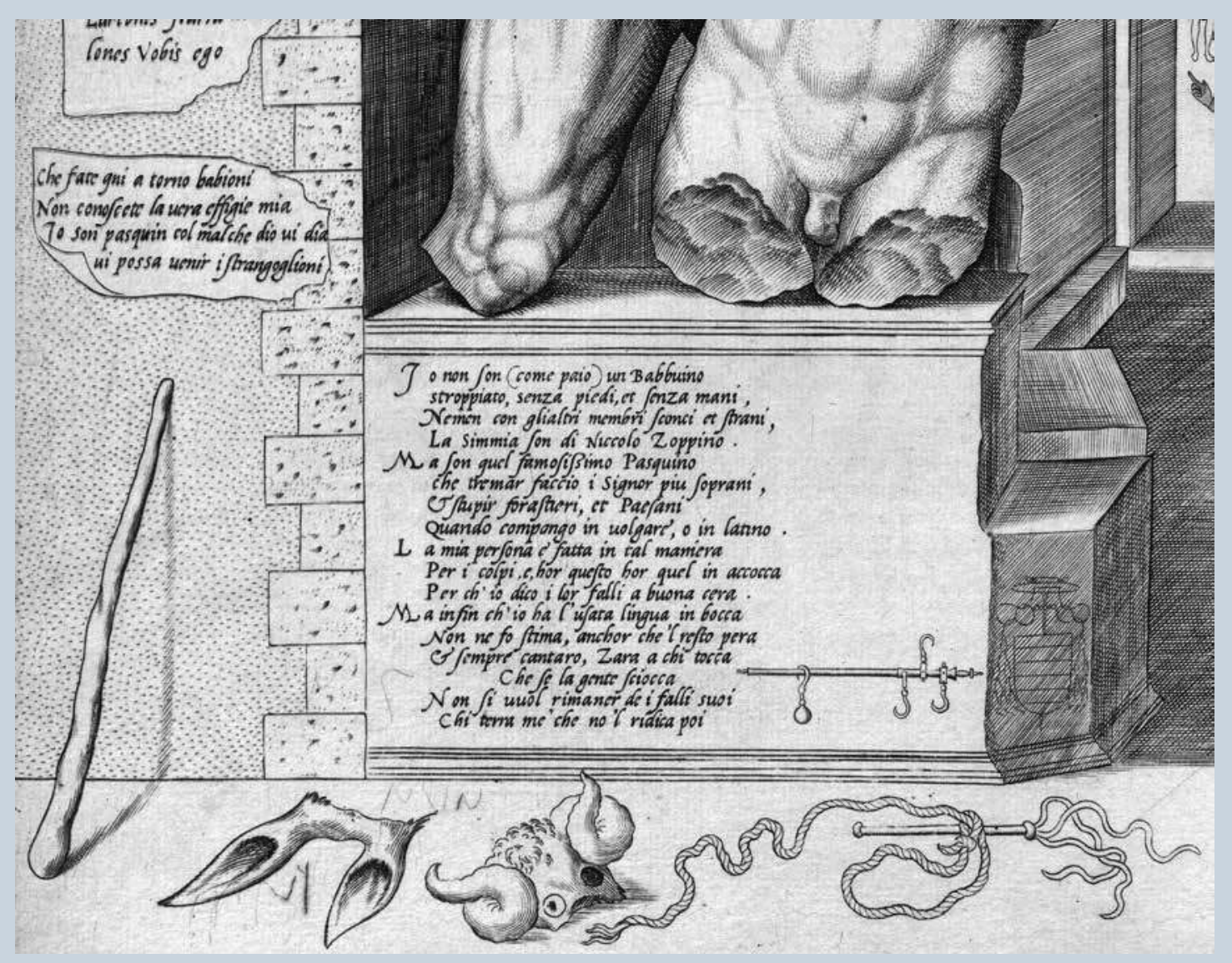

2. Pasquin en gravure (détail). Anonyme, 1550, extrait d'Antoine Lafréry, Speculum Romanae Magnificentiae. 
L'assimilation de Pasquin à une statue miraculeuse maléfique permet qu'on le présente entouré d'ex-voto parodiques, plus librement critiques que les textes. Leur position au pied de la statue suffit à évoquer le dépôt votif, ainsi qu'une tradition picturale de la Renaissance, parfois utilisée pour représenter des ex-voto: les figures de seuil placées dans un espace intermédiaire entre le spectateur et les personnages représentés dans la composition (Golsenne 2015). Ici, le spectateur offre à Pasquin des objets pour lui demander de châtier le pape ou tel cardinal. Ceux-ci matérialisent les armes langagières dont Pasquin se sert pour distribuer les coups et les malheurs plus que les grâces: à la bienveillance de l'image maternelle, il oppose une tête de diable; aux rêves de filiation, qui constituent une part non négligeable des intentions votives, il répond par les cornes du cocu d'un bélier à tête diabolique; aux vertus et aux facultés de la Vierge qui intercède (la tendresse, la miséricorde, l'humilité), il préfère différentes humiliations et punitions: le bonnet d'âne, le bâton, la corde de pendu, qui renvoient aux châtiments des Enfers. La décision de la peine à administrer aux uns et aux autres étant prise après psychostasie pratiquée sur une balance de commerçant placée sur le socle de la statue ${ }^{9}$, qui explicite également l'inscription principale présente sur le socle: Pasquin juge les fautes des uns et des autres, et fait trembler les puissants qu'il destine aux Enfers ${ }^{10}$, au point qu'ils cherchent à le détruire ou à le faire taire. Légèrement postérieure, une autre version de cette même gravure présente Pasquin les yeux fermés et la bouche mutilée. Autour de lui, les papiers affichés sont vides de texte, pour signaler la censure de ces textes pourtant très édulcorés (F. Munoz, Roma, Archivio Fotografico Comunale: statue parlanti, MR 15528, publiée par Marucci, Marzo \& Romano 1986) ${ }^{11}$.

Extrêmement rare à cette époque, la parodie du dispositif votif en contexte religieux confère à l'image une épaisseur de sens qui présente deux avantages sur le texte: elle est immédiate sans être illicite. Immédiate, car elle est immédiatement identifiable; et licite, car elle détourne une forme d'art qu'elle ne critique pas vraiment, à une époque à laquelle la censure est surtout sensible aux textes.

\section{Ex-Voto fecit}

La tradition de la pasquinade politique a la vie dure. C'est, entre autres, aux pieds du Pasquin que l'artiste de rue Ex-Voto a déposé sa « Madonna del Bastone ( «Vierge au Gourdin ») propitiatoire le 29 octobre 2016, pour protester contre la réforme de la Constitution sur laquelle l'Italie a dû se prononcer par référendum le 4 décembre 2016: «Madonna del Bastone, Accorri in soccorso della nostra costituzione » : "Vierge du Bâton, Accours et secours notre constitution», lit-on sur le phylactère que tient la Vierge ${ }^{12}$. Sur l'original (Nicolò Alunno, Madonna del Buon Soccorso, 1482, Rome, Galleria Colonna), Marie menace le Diable qui tente d'arracher un nouveau-né à sa mère. Sur son affiche, Ex-Voto a substitué la Constitution du 27 décembre 1947 à l'enfant, 


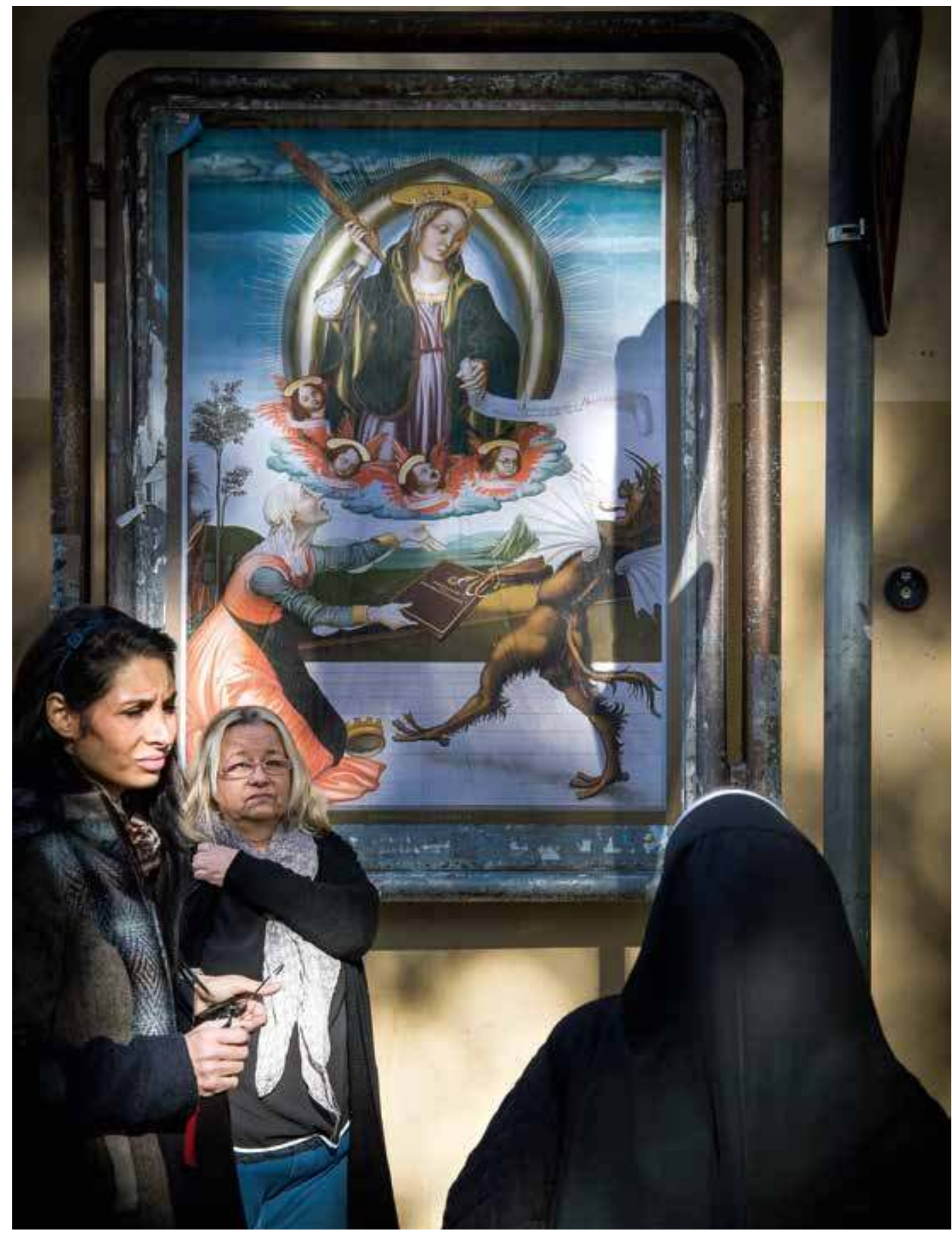

3. Madonna del Bastone Ex-Voto, 29 octobre 2016, Rome, Piazza Testaccio 
et remplacé les chérubins par les portraits des quatre signataires du texte, Alcide De Gasperi, Enrico De Nicola, Giuseppe Grassi et Umberto Terracini.

Depuis une dizaine d'années, Ex-Voto a réalisé une soixantaine de Vierges miraculeuses parodiques, parfois satiriques, qui se jouent de la reproductibilité: disponibles en ligne ${ }^{13}$, ces images multiples sont potentiellement aussi nombreuses que les images de dévotion que le touriste religieux se procure par centaines dans les officines du Borgo Pio. Elles représentent la «spécialité» de chaque Vierge miraculeuse (le Socialisme, l'Anarchie, la Famille, le Mensonge, la Loterie, les Mauvaises Odeurs...) ainsi qu'une inscription votive propitiatoire. Faux ex-voto, les affiches ne contreviennent pas tant que cela aux traditionnelles spécialités des Vierges miraculeuses qui touchent l'ensemble des champs possibles de la vie, les fameux «amour; travail; santé » de nos horoscopes modernes. D'où le jeu serré qu'instaure Ex-Voto avec le code du dispositif votif, dans la pratique catholique italienne. D'où le succès de ses installations auprès des publics, des médias et des critiques (Cucchiarelli 2018, Dal Lago \& Giordano 2018).

Examinons de plus près la facture des affiches, qui opèrent une synthèse entre deux traditions figuratives bien identifiées: l'image mariale iconique, à proximité de laquelle sont déposés les ex-voto, et le tableautin votif historié. L'artiste maintient l'apparence générale de la Vierge à l'Enfant, et notamment ses dimensions dans l'image, rendant possible un culte. Au tableautin votif historié, il emprunte les inscriptions ainsi que quelques éléments iconographiques qui ne viennent pas perturber la structure iconique de l'image mariale, étant habituellement rejetés en bas du format: ainsi de la Constitution, des moustaches et des lunettes de certains signataires du texte. Cette double tradition ressort également des propos de l'artiste, qui brouille - volontairement - les limites entre les deux types d'images: Ex-Voto signe «Ex-Voto fecit» parce qu'il considère que son geste est votif, et que ses affiches constituent des ex-voto; mais il se dit également «Smadonnaro posterista» (« défaiseur de Madones postériste»), reprenant le terme de Madonnaro (peintre de Vierges à l'Enfant) associé aux origines du street art italien que l'on fait souvent remonter au $\mathrm{XVI}^{\mathrm{e}}$ siècle.

La fabrique du faux fonctionne ainsi. Ex-Voto emprunte des Vierges à l'Enfant à l'histoire de l'art, avec une prédilection pour la Renaissance italienne. Il les retouche ensuite à l'ordinateur, insérant ou modifiant des inscriptions propitiatoires, changeant des figures ou des détails iconographiques, effectuant parfois des collages numériques entre plusieurs œuvres, éventuellement de plusieurs époques, afin de leur donner une tournure parodique liée à l'actualité politique et sociétale au sens large: citons ainsi la Splendida Madre della Resistenza ("Splendide Mère de la Résistance »), la Santa Madonna dell'Autoscatto ( Sainte Vierge du Selfie »), l'Immacolata Contracezzione («L'immaculée Contraception») ou encore la Madonna delle Buche ( La Vierge des nids de poule». Souvent raillés par Pasquin, les «nids de poule» (buche) de Rome sont devenus le symbole des carences de la municipalité). Puis il les tire en affiches de dimensions variées et les place en ex-voto, comme des Madones de rue, dans des lieux et à des dates en rapport étroit avec le sujet représenté. Souvent installées dans des édicules, niches, lieux qui «font autel», les Vierges sont entourées de premiers objets cultuels, fleurs et bougies de neuvaines à l'image de la Vierge ou de «saints», voire médailles à l'effigie de la Vierge célébrée, les transformant 


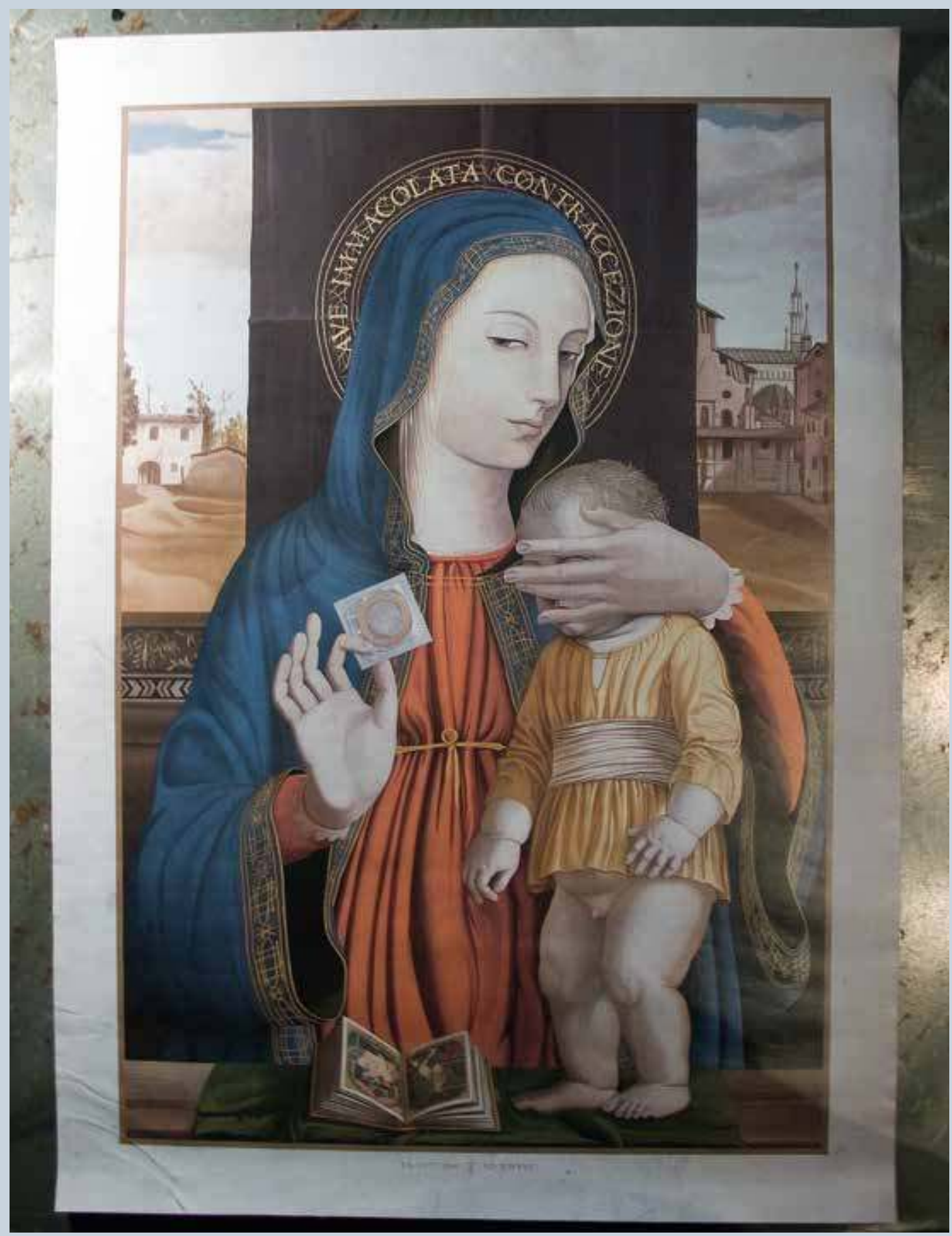

Immacolata Contraccezione Ex-Voto, 8 déc. 2016 
en installations de rues. Rendu visible, le culte est lancé et soumis aux réactions - nombreuses et radicales - des habitants et des passants. De véritables rapports de force s'instaurent alors entre d'un côté, Ex-Voto en personne, les amateurs éclairés, les partisans du culte et, de l'autre, les partisans de l'ordre, les opposants au blasphème, les opposants politiques et les services de nettoyage municipal. Certains malmènent les affiches, d'autres commencent à leur rendre un culte, brouillant la frontière entre parodie et original, entre reconnaissance du geste artistique et culte ou respect réflexe pour l'image de la Vierge à l'Enfant. Parmi les amateurs et les dévots, certains commencent à intercéder auprès des services d'entretien ou des autorités laïques, et parfois même religieuses, pour qu'on n'enlève pas les Madones, ou pour qu'on les remette. En voici quelques exemples.

\section{La Beata Vergine degli Scolari ("Bienheureuse Vierge des Écoliers ")}

La Beata Vergine degli Scolari date d'octobre 2012 et est réapparue au moment du besoin, en mai $2015^{14}$. Ex-Voto a modifié la Madonna di Crevole de Duccio (1283-1284, Sienne, Musée de l'œuvre de la cathédrale). Gardant son corps d'enfant, Jésus a désormais une tête d'âne, comme si l'école allait devenir le pays des Balocchi de Collodi (Pinocchio, chap. XXXI-XXXII). Quant à la Vierge à mi-corps de Duccio, elle est devenue une Vierge en pieds piétinant le serpent de l'ignorance. Devant ses jambes, deux angelots présentent un livre ouvert sur la requête votive suivante: "Beata Vergine degli Scolari, aiuta i nostri figli a non diventare somari» ("Bienheureuse Vierge des Écoliers, aide nos enfants à ne pas devenir des ânes»). Un détourage blanc simule l'apparition de personnages qu'on croirait en lévitation.

Cette Vierge fonctionne particulièrement bien dans certains lieux. En 2015, Ex-Voto l'a placée Viale Trastevere, juste en face du Ministère de la Pubblica Istruzione (actuel MIUR), afin de protester contre la réforme dite de la «Buona Scuola» (la «Bonne École») votée le 13 juillet 2015 à l'initiative du gouvernement Renzi. Mais elle n'est pas seulement située en face du Ministère: elle jouxte également l'un des murs votifs de Rome, celui de la Madonna degli Orfani (la Vierge des Orphelins), une Vierge en terre cuite de style néorenaissant réalisée en 1944 par le ferrarais Arrigo Minerbi (1881-1960), vénérée depuis et entourée de nombreuses inscriptions votives. S'instaurent ainsi une concurrence entre les deux Vierges, et des jeux de continuité entre vrais et faux ex-voto, entre plaques votives célébrant les succès des écoliers et graffiti très éphémères: un «P.G.R. PORCO GESÜ RANDAGIO » («P.G.R. Cochon de Jésus vagabond») a détourné à son tour le traditionnel «Per Grazia Ricevuta», répondant à l'installation d'Ex-Voto et à une plaque votive évoquant le cheminement de l'écolier. Quelques jours après l'installation de l'affiche, la tête d'âne de l'Enfant avait été grattée. Le 28 avril 2018, le P.G.R., avait disparu, recouvert par la peinture, tandis qu'on devinait encore la silhouette et quelques détails de l'affiche plusieurs fois déchirée. 


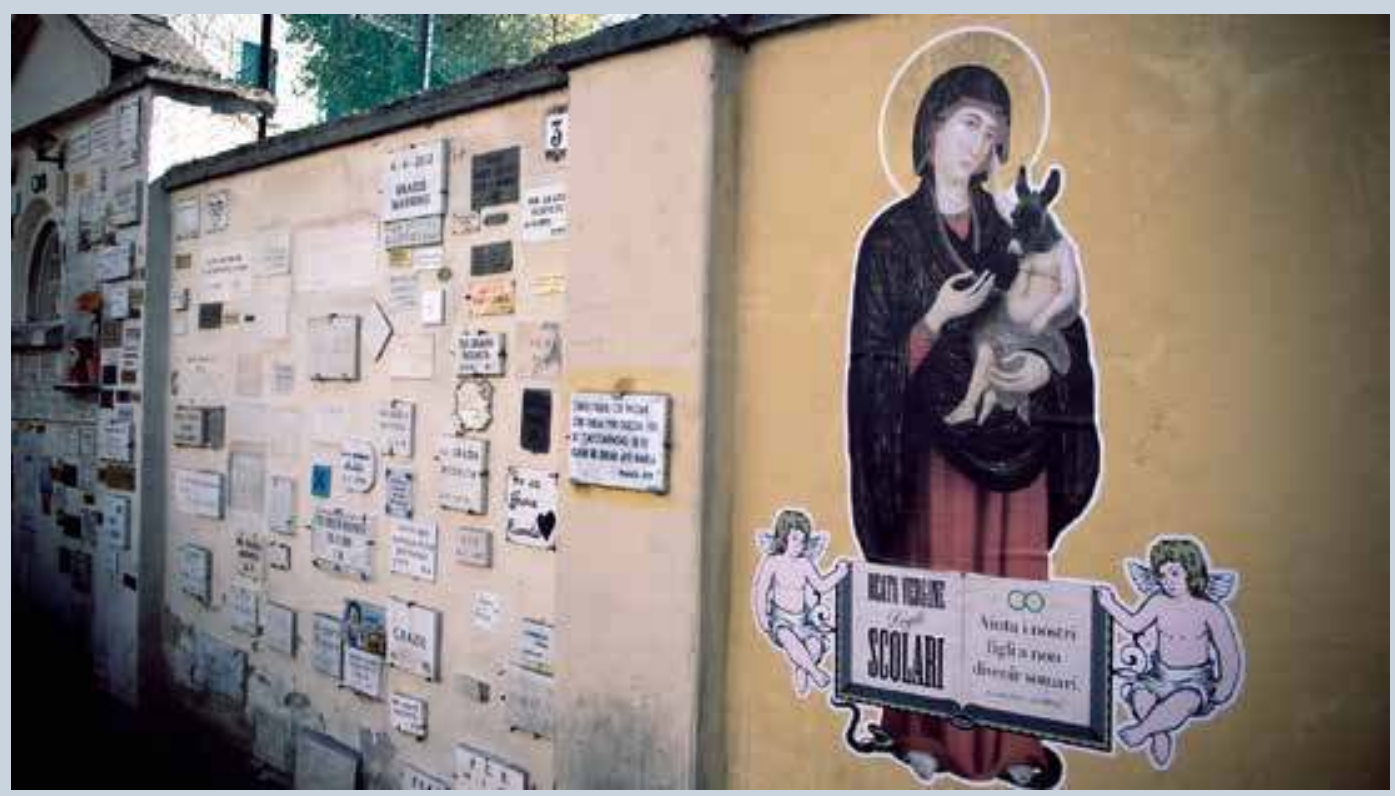

5. La Beata Vergine degli Scolari et le mur votif de Viale Trastevere (Rome). Ex-Voto, mai 2015
Tête d'âne grattée. Ex-Voto, Beata Vergine degli Scolari à Viale Trastevere, mai 2015 , détail.

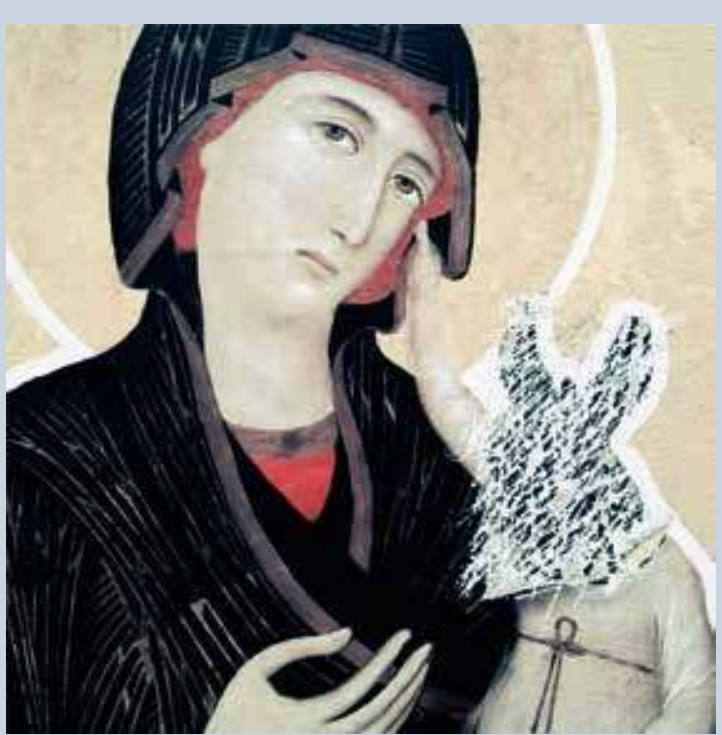




\section{Nostra Signora der Futteball ("Notre Dame du Ballon rond ")}

Ex-Voto travaillant à Testaccio (un quartier populaire en voie de gentrification) et ses travaux étant rythmés par le calendrier, on ne s'étonnera pas que Nostra Signora der Futteball (rigoureusement vénérée en dialecte romain) fasse de fréquentes apparitions dans le quartier historique de l'équipe de Rome, la Roma, créée en 1927. Les demandes d'intercession se multiplient logiquement à l'approche des matchs. Inspirée d'une représentation de l'Immaculée Conception, Marie se dresse sur un ballon en cuir vintage si gros qu'il met en fuite le diable rouge et noir du Milan, le serpent de l'Inter et le zèbre de la Juventus - trois des meilleures équipes de la Série A. Bien placé à la droite de la Vierge, l'aigle votif de la Roma ouvre les bras comme le font les joueurs qui viennent de mar-

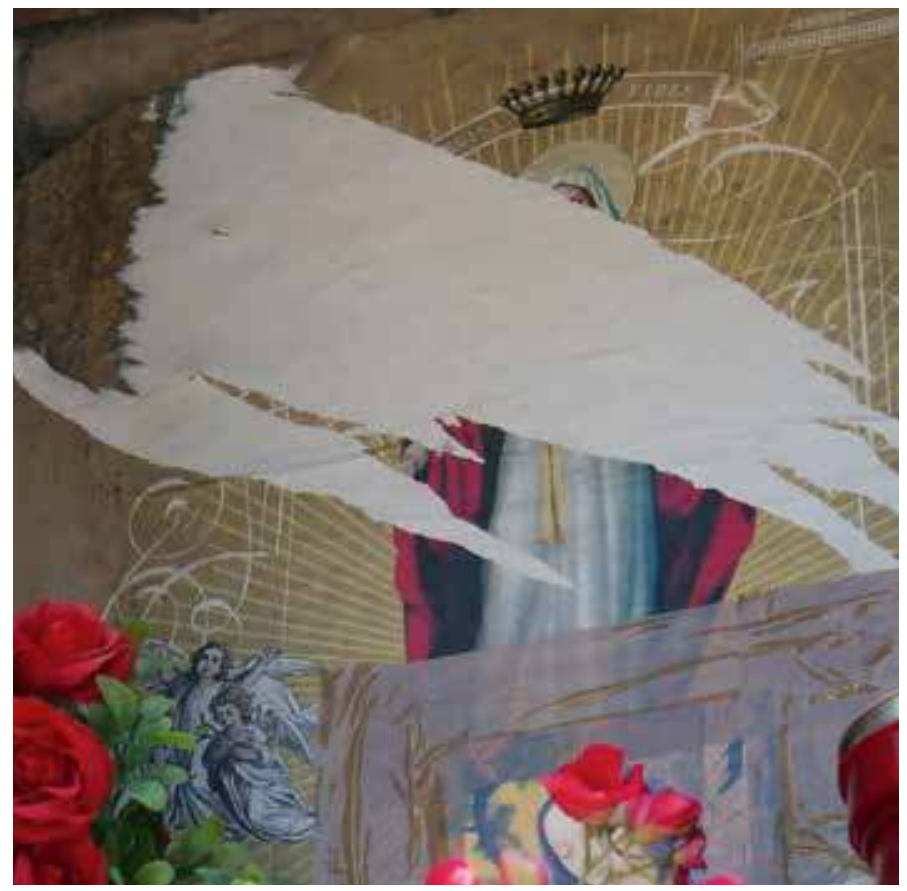
quer pour remercier la Vierge sauroctone. Dans la première version de l'affiche (2011), sous le Ballon-Monde, deux joueurs de la Roma des origines présentent leur requête, un genou à terre: «Nostra Signora der Futteball, Facce la grazia: aridacce er campo e aridacce 'na squadra» ("Notre Dame du Ballon rond, fais-nous cette grâce: rends-nous notre stade et une équipe digne de ce nom »). Car comme la Roma, Nostra Signora der Futtball est étroitement liée au quartier, où elle signale l'emplacement du premier stade de l'équipe (le fameux campo mentionné par l'inscription). Ce quartier historique accueille la Madone depuis février 2011. Plus précisément, la petite affiche $(36 \times 48 \mathrm{~cm})$ a trouvé place dans un compteur électrique désaffecté qui lui sert d'édicule afin d'y déposer des dons votifs: fleurs, neuvaines portant l'image de joueurs sanctifiés par le temps, petites figurines de joueurs en plastique etc. De l'aveu même de l'artiste, cette Madone de quartier, qui joue sur les fiertés et sur les gloires locales, est celle qui dure le plus et qui a le mieux pris. Rien d'étonnant à cela, tant les sanctuaires d'Italie regorgent d'objets votifs liés au sport et en particulier au foot. Devant Nostra Signora der Futtball, des mains inconnues déposent régulièrement des fleurs, des lumières votives, des images dévotionnelles en italien: tiffosi acharnés, romains spirituels ou dévots désormais habitués à l'image du quartier? En 2016, des images de la Vierge accompagnées d'inscriptions en cyrillique sont apparues: hommage à Luciano Spalletti, alors entraîneur de la Roma, et ancien entraîneur du Zenit de Moscou, ainsi que l'artiste l'avait alors suggéré? Pèlerins orthodoxes égarés à Testaccio, ne pouvant déchiffrer

\footnotetext{
7. Le culte de Nostra Signora der Futteball. Ex-Voto, Rome, Campo Testaccio, 18 mars 2016

8. Nostra Signora der Futteball, Новый завет (Nouveau Testament en russe) et Padre Pio. Ex-Voto, Rome, Campo Testaccio,

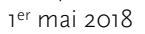


l'inscription en dialecte romain? Ou communauté locale qui s'est emparée de la Vierge? À en juger par la situation actuelle, il semblerait que la dernière solution soit la plus probable. Nostra Signora ayant subi les injures du temps, Ex-Voto fecit a renouvelé l'affiche devant laquelle des mains inconnues ont déposé des fleurs, des neuvaines de Padre Pio ainsi qu'un Nouveau Testament en cyrillique.

\section{Generosa Madre dell'Accoglienza ("Bonne Mère de l'Hospitalité ")}

Créée en avril 2016, la Generosa Madre dell'Accoglienza a fait l'objet de réactions particulièrement violentes en raison de son emplacement, de ses dimensions et bien sûr de son sujet. Elle a été installée au Lido d'Ostie les 7 et 8 mai 2016 dans le cadre de l'exposition UrbanArea-A Scena Aperta, organisée par le collectif aDNA. Plus précisément, l'affiche a été collée dans la niche d'un centre social située sur rue, en faisant une Madone aux dimensions d'un tableau d'autel ou d'une publicité moderne (2,30 x 3,30 m), juste sous le nom du centre écrit en gigantesques caractères rouges: AFFABULAZIONE. Le sujet justifiait les dimensions: la Generosa Madre dell'Accoglienza venait rappeler que la Charité (Agapè), vertu théologale énoncée par Paul dans sa première épître aux Corinthiens (I Co 13, 13), ordonne aux chrétiens l'amour de leur prochain, et donc l'accueil des immigrés, d'où l'idée de la faire voyager jusqu'à Lampedusa (où elle n'est pas encore arrivée).

Pour composer sa Vierge critique et sans doute plus votive que d'autres, Ex-Voto a emprunté sa Charité entourée de cinq enfants blancs, potelés et plutôt blonds (1878, Northampton, Mass, Smith College Museum of Art) au peintre académique William-Adolphe Bouguereau (1825-1905). Après avoir coiffé la Charité d'une couronne, pour en faire une Vierge, et après

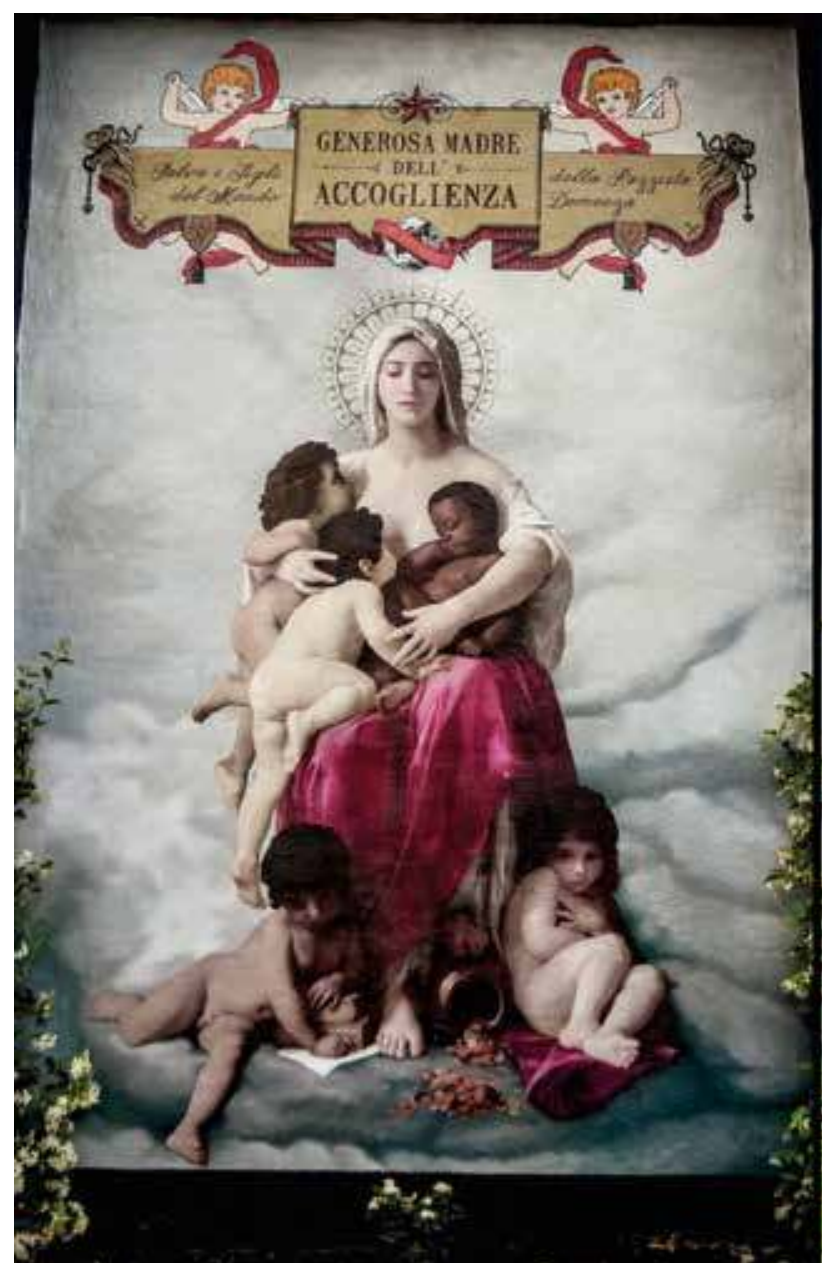


l'avoir placée dans les cieux, il a notamment assombri les cheveux des enfants et surtout la peau de celui qui dort contre le sein de la Vierge, au point de le rendre noir. Un des enfants tente quant à lui de récupérer des papiers coincés sous le pied de la Bonne Mère. En haut, deux angelots stylisés présentent une requête lisible par le spectateur: «Generosa Madre dell'Accoglienza, Salva i figli del Mondo della Razzista Demenza» («Bonne Mère de l'Hospitalité, Sauve les enfants du Monde de la Raciste Insanité»). De chaque côté de la Vierge, l'artiste a installé deux jasmins grimpants, verticaux et bien blancs.

Placer cette affiche à Ostie n'était pas anodin. Le littoral du Latium constitue en effet l'un des bastions du parti néo-fasciste CasaPound, ainsi que l'ont encore démontré les svastikas et les croix celtiques graffitées sur le centre social en 2017, à Pâques, ou les récentes élections de

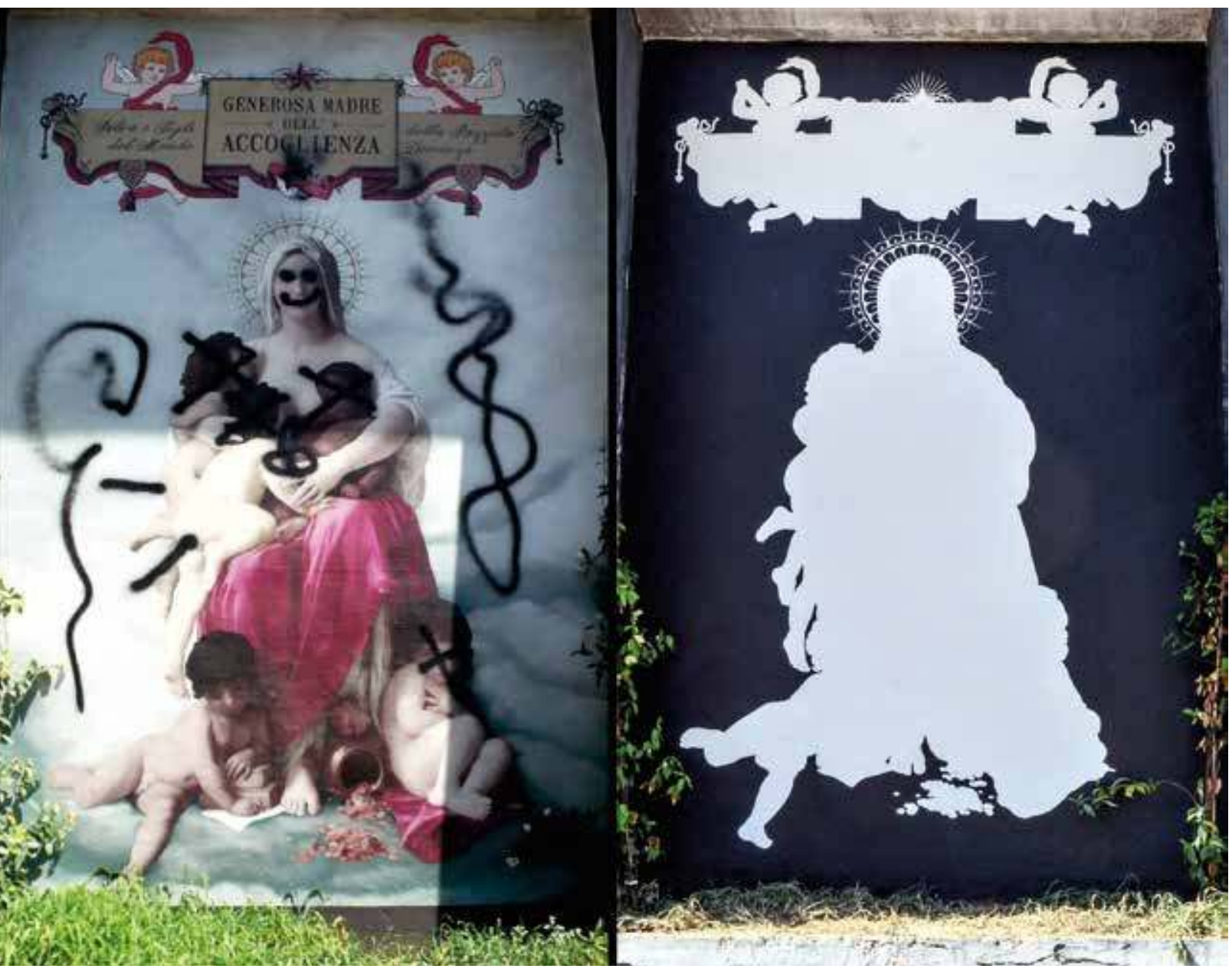

9 \& suivante. La Generosa Madre dell'Accoglienza à Ostie en 2016 Ex-Voto, mai-juillet 2016, Ostia Lido, Centro Sociale Affabulazione. 


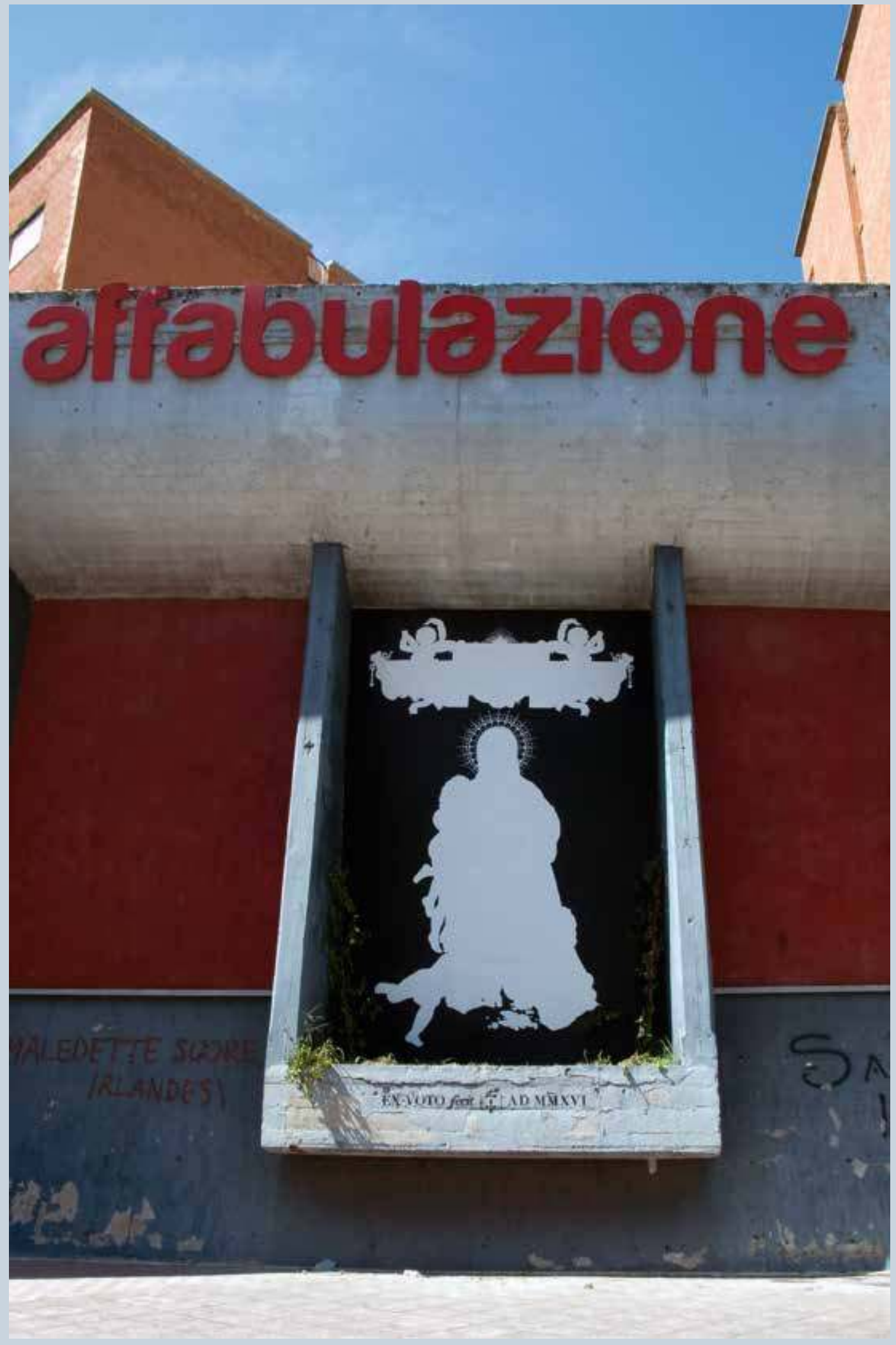


2017. Les habitants du quartier ont immédiatement adopté l'œuvre, et les dévots, et surtout les dévotes, ont commencé à rendre un véritable culte à cette gigantesque Vierge de l'immigration, en lui portant notamment des fleurs et en lui adressant des prières. L'affiche a résisté deux mois, avant d'être barbouillée de noir (couleur des fasci), notamment sur le visage de la Vierge, et avant que des croix soient apposées sur les figures des enfants, également affublés de queues suggérant l'animalité des immigrants. Les habitants du quartier ne se sont pas résignés, et sont allés jusqu'à solliciter l'évêque, qui a intercédé pour que la Vierge, qui aurait été bénie au terme d'une procession partie de l'église (!), reprenne sa place. Lauteur a finalement remis en place une silhouette - au grand dam des dévots et dévotes.

Le remploi du dispositif votif, a fortiori de la forme élémentaire de l'ex-voto, notamment les morceaux anatomiques, est massif et extrêmement varié dans l'Italie contemporaine: des gros cœurs enflammés piqués sur les collections Dolce e Gabbana 2015 aux complexes tissages votifs de l'artiste romaine Lea Contestabile (1949-). Stylistes, dessinateurs (Buzzati 1972), caricaturistes, artistes de tous secteurs s'en sont emparés, parfois avec talent, à des fins très différentes. Faut-il y voir une omniprésence des formes religieuses, réutilisées à la façon d'un lexique disloqué et indirect, et parfois d'une référence gratuite? Dans certains cas, comme celui de la mode, peutêtre. Mais force est de constater qu'il s'agit souvent de références directes autant qu'étayées. Or la référence directe à l'art religieux dans l'art contemporain occidental a pu être qualifiée de sporadique (Grenier 2003). Elle ne l'est pas dans les terres de religion encore active, et elle n'est certainement pas gratuite si l'on considère en priorité les formes artistiques destinées à faire réagir des destinataires parfois contraints, comme le street art ou la caricature, plutôt que les œuvres pensées pour des publics restreints et intentionnels (le lecteur du livre illustré, le visiteur de la galerie d'art etc.). En dernier lieu, cette référence se présente avant tout comme une langue durablement vivante, qui s'inscrit dans l'histoire longue de ces dispositifs, des dispositifs (ou des formes, celle de l'organe par exemple) dont on a justement dit qu'ils évoluaient peu (DidiHuberman 2006) : en 1550 ou en 2018, l'identification du dispositif votif est immédiate à Rome, comme elle l'est en Espagne ou au Mexique. Et elle est efficace, puisqu'elle suscite effectivement des sentiments mêlés et des réactions de différents types: censure, dommages, hommages.

\section{Notes}

1. J'appelle dispositif une série de codes déterminant la structure de l'image: hauteur du socle, rapport entre les ex-voto et l'image miraculeuse, frontalité de l'image, cadrage des personnages. Ce mot permet d'éviter celui de style ou, plus précis et ambigu, celui de forme. Dans le cas de l'ex-voto catholique,

la durabilité des dispositifs est infiniment supérieure à celle des styles.

2. L'édition de référence des pasquinades présente différents frontispices des Carmina apposita Pasquillo, ces recueils annuels de pasquinades déguisées et fêtées pour la saint Marc. On l'y voit transformé en 
douze personnages différents: Marucci, Marzo \& Romano 1983, t. II, Tav. XXX-XLI.

3. À la vocation politique précoce de Pasquin, qui marque les rapports de force internes à la Ville, il faut ajouter une veine gaillarde depuis l'Arétin (1492-1556), dans la tradition de l'épigramme antique, que nous laisserons de côté.

4. Marucci, Marzo \& Romano 1986, t. 1, 136: 108109: "Ad Leonem V de Martino Luthero Pasquinus in Cardinales generales tres. »

5. Le modèle michelangelesque est évident. Il l'est dans toutes les gravures du Speculum représentant des statues antiques: les Dioscures, le Marforio, dont l'anatomie est traitée comme celle de la gravure du Christ de la Minerve (Rome, Santa Maria sopra Minerva) de Michel-Ange.

6. Parmi celles-ci, la faculté de se transformer: «Pasquin tu fuste e sarai semper un paz' / Ti sai in ogni forma trasformare / Se ti voi alli donne grato fare / Per che non ti tra'fformi in t'un Caz'. » ("Pasquin tu fus et toujours fou seras / Toi qui sais prendre toutes les formes / Si tu veux faire plaisir aux femmes / Transforme-toi en Pha'. »

7. Le Babbuino étant la statue parlante la plus triviale.

8. «Lussones scurra nebu/lones vobis ego.» ("Je suis votre bouffon, / garnements qui aimez rire. »).

9. En 1580, Annibal Carrache représente ainsi la balance de sa Boucherie (Oxford, Christ Church).

10. «Io non son (come paio) un Babbuino / Stroppiato, senza piedi, et senza mani, / Nemen con gli altri membri sconci et strani, / La Simmia son di Niccolo Zoppino. / Ma Son quel famosissimo Pasquino / Che tremar faccio i Signor piu soprani, / Et stupir forastieri, et Paesani / Quando compongo in volgare', o in latino. / La mia persona è fata in tal maniera / Per i colpi, e, hor questo hor quel in accocca / Per ch'io dico i lor falli a buona cera. / Ma infin ch'io ha l'usata lingua in bocca / Non ne fo stima, anchor che 'I resto pera / E sempre cantaro, Zara a chi tocca I Che se la gente sciocca / Non si vuol rimaner de i falli suoi / Chi terra me' che no'l ridica poi. » ("Je ne suis pas (comme je le semble), un Babouin, / Difforme, sans pieds, sans mains, / Et avec mes membres obscènes et curieux, / Je ne suis pas le Singe de Niccolò Boiteux. / Mais je suis ce très illustre Pasquin, / Qui fait trembler les souverains, / S'émerveiller les voyageurs et mes concitoyens / Quand je compose en vulgaire ou en latin. / Si ma personne est ainsi faite / C'est pour les coups, dont les uns et les autres m'agacent / Quand je leurs dis leurs fautes en pleine face. / Mais tant que j'ai ma vieille langue en bouche / Ça m'indiffère, puisque le reste passera / Et moi je chanterai toujours, tant pis pour celui sur qui ça tombera. / Et si les gens benêts / Veulent oublier leurs méfaits / Malin qui me gardera moi de les colporter. »

11. La fonction d'intercesseur de Pasquin ne s'est pas démentie avec le temps, et dure depuis 500 ans. Elle est indissociable de l'histoire de la poésie italienne et de la poésie romaine en langue vernaculaire, particulièrement gouailleuse. La réputation du plus célèbre poète romain, Gioacchino Belli (17911863), ou, plus tard, de Trilussa (1873-1950), s'est construite autour de Pasquin (Anonimo 1972), depuis «copié » et reproduit sur le monument à Belli, inauguré en 1913. Pasquin y est entouré du «popolo romano» occupé à l'entendre (Gigli 2014). Depuis la restauration de 2009 , les pasquinades doivent cependant être accrochées sur un panneau de plexiglas placé à côté de la statue. Et celles qui bravent l'interdit sont tôt enlevées ou déplacées. Il s'agit soit d'une tentative de censure, soit d'un excès de précaution, le socle de Pasquin ayant une valeur historique et non artistique.

12. Entretien réalisé avec l'artiste le 16 avril 2018.

13. Sur Artflakes, en plusieurs dimensions. Pour favoriser la reproductibilité et la multiplicité de ses affiches, l'artiste a volontairement maintenu des prix très bas, évitant le marché et les collectionneurs.

14. Il existe un sanctuaire de la Madonna degli Scolari à Laino Catsello, en Calabre.

\section{I'auteure}

Anne Lepoittevin est maître de conférences en histoire de l'art moderne (Sorbonne Université). Ses recherches portent sur l'art religieux italien à l'époque moderne, notamment sur la statuaire (Sacri Monti) et sur les objets de dévotion (ex-voto, Agnus Dei). 


\section{Iconographie}

Image d'ouverture. Pasquin à Rome aujourd'hui. (C) Rémi Jouan.

1. Extrait d'Antoine Lafréry, Speculum Romanae Magnificentiae, v. 2, pl. 102, 40 × 28 cm. (C) Met, Metropolitan Museum of Art, Prints, 41.72(2.102).

2. Extrait d'Antoine Lafréry, Speculum Romanae Magnificentiae, v. 2, pl. 102, 40 x 28 cm. (C) Met, Metropolitan Museum of Art, Prints, 41.72(2.102).

3. Madonna del Bastone. Ex-Voto, 29 octobre 2016, Rome, Piazza Testaccio, 100 x $140 \mathrm{~cm}$. (C) Valentino Bonacquisti. 4. Immacolata Contraccezione. Ex-Voto, 8 décembre 2016, 70 x $100 \mathrm{~cm}$. (C) Ex-Voto.

\section{Références}

Anonyme, 1972 La Satira a Roma. Da Pasquino a Trilussa. Rome: EDI.

Buzzati, D. 1971 I Miracoli di Val Morel. Milan: Garzanti.

Catelli, N. 2008 Scherzar coi santi: prospettive comiche sul Sacco di Roma. Parme: Uninova.

Chastel, A. 1984 Le sac de Rome, 1527. Du premier maniérisme à la Contre-Réforme. Paris: Gallimard.

Cucchiarelli, C. 2018 Quello che i muri dicono. Guidonia: Jacobelli Editore.

Dal Lago, A. \& S. Giordano 2018 dir. Sporcare i muri. Graffiti, decoro, proprietà privata. Rome: Derrive Approdi.

Damianaki, C. \& A. Romano 2014 Pasquin, Lord of Satire, and his Disciples in $\mathrm{XVI}^{\text {th }}$ century Struggles for Religious and Political Reform. London: The Warburg Institute / Edizioni di Storia e Letteratura.

Didi-Huberman, G. 2006 Ex-voto: image, organe, temps. Paris: Bayard.

Gigli, L. 2014 « Il Monumento a Giuseppe Gioachino Belli racconta. Ascoltiamolo parlare », Strenna dei Romanisti $75: 215-230$.

Golsenne, T. 2015 «Carlo Crivelli. Portrait of the Artist as a Cucumber», in S. J. Campbell dir., Ornament and Illusion. Carlo Crivelli of Venice, Londres, Paul Holberton Publishing: 78-93.

Grenier, C. 2003 L'art contemporain est-il chrétien? Nîmes: Jacqueline Chambon.

\section{Pour citer l'article}

Lepoittevin, A. 2018 «Pastiches votifs à Rome. Du Pasquin à Ex voto fecit», TechniquesE Culture 70 «Matérialiser les désirs. Techniques votives», p. 220-239.
5. La Beata Vergine degli Scolari et le mur votif de Viale Trastevere (Rome), mai 2015, 145 x $100 \mathrm{~cm}$ @ Ex-Voto.

6. Tête d'âne grattée. Beata Vergine degli Scolari à Viale Trastevere, mai 2015, détail. (c) Ex-Voto.

7. Le culte de Nostra Signora der Futteball. Rome, Campo Testaccio, 18 mars 2016, 41 x $55 \mathrm{~cm}$. (C) Ex-Voto.

8. Nostra Signora der Futteball, Новый Завет (Nouveau Testament en russe) et Padre Pio. Rome, Campo Testaccio, 1er mai 2018. (C) Ex-Voto.

9. La Generosa Madre dell'Accoglienza à Ostie, mai-juillet 2016, 230 x 330 cm, Ostia Lido, Centro Sociale Affabulazione. (C) Ex-Voto.

Karmon, D. 2008 «Printing and Protecting Ancient Remains in the Speculum Romanae Magnificentiae», in R. Zorach dir. The Virtual Tourist in Renaissance Rome. Printing and Collecting the Speculum Romanae Magnificentiae. Rome: Salerno Editrice: 37-55.

Lastriaoli, C. 2014 «La conversione di Pasquino : la satira anticlericale e riformata europea», in C. Damianaki \& A. Romano dir. Pasquin, Lord of Satire, and his Disciples in XVI ${ }^{\text {th }}$ century Struggles for Religious and Political Reform. London: The Warburg Institute / Edizioni di Storia e Letteratura: 3-15.

Marucci, V., Marzo, A. \& A. Romano dir. 1983 Pasquinate Romane del Cinquecento. Rome: Salerno Editrice, 2 tomes.

Michel d'Annoville, C. \&Y. Rivière dir. 2016 Faire parler et faire taire les statues. De l'invention de l'écriture à l'usage de l'explosif. Rome: EFR

Parshall, P. 2006 «Antonio Lafreri's "Speculum Romanae Magnificentiae" », Print Quarterly 23: 3-28.

Sansterre, J.-M. 2016 «Les Images parlantes des catholiques, du Moyen Âge aux Temps modernes, et la polémique protestante ( $\mathrm{XvI}^{\mathrm{e}}$-XvII ${ }^{\mathrm{e}}$ siècles). Une première approche », in C. Michel d'Annoville \&Y. Rivière dir. Faire parler et faire taire les statues. De l'invention de l'écriture à l'usage de l'explosif. Rome: EFR: 59-77.

Zorach, R. 2008 The Virtual Tourist in Renaissance Rome. Printing and Collecting the Speculum Romanae Magnificentiae. Chicago: University of Chicago Press. 